\title{
Etude de la lysotypie \\ et de la résistance aux bactériophages chez les streptocoques lactiques mésophiles
}

\author{
par \\ Bernadette BOISSONNET, J. Y. BOZIO, C. BRIERE \\ Marie-Claude LECLERC et J.P. LARPENT*
}

L'utilisation des levains dans l'industrie laitière nécessite que les souches qui les composent soient judicieusement associées pour assurer l'acidification du caillé et la création d'arômes et pour éviter les accidents phagiques. Les espèces les plus importantes sont: Streptococcus lactis, diacetylactis et cremoris.

De très nombreux auteurs se sont intéressés à la lysotypie des streptocoques lactiques dans le but d'organiser scientifiquement des rotations de souches dans l'industrie laitière $[2,6,8,4,5,10,11,12]$.

Nous avons tenté d'établir la lysotypie des 3 espèces de Streptococcus mésophiles en essayant de regrouper les phages selon leur affinité d'hôte.

\section{MATERIEL ET METHODES}

164 souches bactériennes provenant de collection ou de l'industrie ont été lysotypées vis-à-vis de 79 types de bactériophages (phages de $S$. cremoris, $S$. lactis et de $S$. diacetylactis). 3 tests de lysotypie ont été réalisés à un mois d'intervalle. Les souches sont conservées lyophilisées à $+4^{\circ} \mathrm{C}$. Les dénombrements phagiques sont réalisés sur milieu de Mac Kay [9] gélosé. Le calcul du "Routine test dilution » a permis de définir le titre de la suspension à utiliser $[7,8]$.

Les stocks de phages "présumés» provenant de lactosérum et de lait ont été centrifugés pendant 15 minutes à $3000 \mathrm{~g}$. Le surnageant a été recueilli pour les tests ultérieurs réalisés sur 15 cultures de streptocoques lactiques mésophiles d'origine industrielle.

* Laboratoire de Microbiologie, 4, rue Ledru - 63000 Clermont-Ferrand. 


\section{RESULTATS}

$1^{\circ}$ Pour la lecture des résultats, les conventions suivantes ont été établies : \pm : de 1 à 50 plages/goutte ;

+ : de 50 à 100 plages/goutte ;

++ : 100 plages/goutte.

Par une analyse des similitudes de sensibilité des souches, réalisée à l'ordinateur par la méthode des "nuées dynamiques » [3], il est possible de dégager 10 groupes de lysotypie regroupant respectivement $6,4,9,12,5,12,11,10,15$ et 4 souches.

5 souches se sont révélées très sensibles aux bactériophages disponibles dans notre laboratoire, donc susceptibles ultérieurement de servir de souches indicatrices pour dépister en laiterie la présence éventuellement dangereuse de bactériophages (fig. 1 et 2). Enfin, 54 souches sont difficilement typables car elles ne présentent qu'une seule réaction avec leur phage homologue ou des réactions positives de lysotypie dispersées parmi les différents groupes. Enfin 16 souches, 2 par 2, se sont révélées identiques quant à leur sensibilité aux bactériophages. Leur propriété biochimique étant très voisine, le test de lysotypie est donc venu confirmer leur identité.

Un phage s'est révélé peu spécifique, car il attaque indifféremment $S$. lactis, $S$. diacetylactis et quelquefois $S$. cremoris. Ce virus est donc intéressant pour tester la résistance de certaines souches bactériennes.

Il ne semble pas y avoir d'interférence entre les phages de $S$. cremoris et les souches de $S$. diacetylactis. Les phages homologues de $S$. lactis attaquent apparemment d'une manière comparable les $S$. cremoris et $S$. diacetylactis. Les phages homologues de $S$. diacetylactis attaquent $S$. lactis et $S$. diacetylactis, mais sont inefficaces sur $S$. cremoris.

2。 105 sérums de fromageries diverses ont été testés. Seuls, 36 sérums sur 105 sont inactifs vis-à-vis de 15 cultures de streptocoques lactiques mésophiles d'origine industrielle. Une culture a été attaquée par 4 sérums différents, une autre par 36 (tableau 1).

Certains sérums ont un grand spectre d'action. Les bactériophages correspondent à ceux étudiés par Leclerc [4] et sont capables d'attaquer un grand nombre de souches bactériennes.

Sur 97 réactions positives à $32^{\circ} \mathrm{C}, 27$ sont de type muralysine (ou éventuellement dues à un inhibiteur présent dans le lait: antibiotique ou bactéricide, ou inhibiteurs divers) autrement dit, la lyse des bactéries n'est pas provoquée par le déroulement du cycle lytique viral, mais par l'action d'une grande quantité de particules phagiques qui, sans pénétrer dans la bactérie, détruisent celle-ci de 


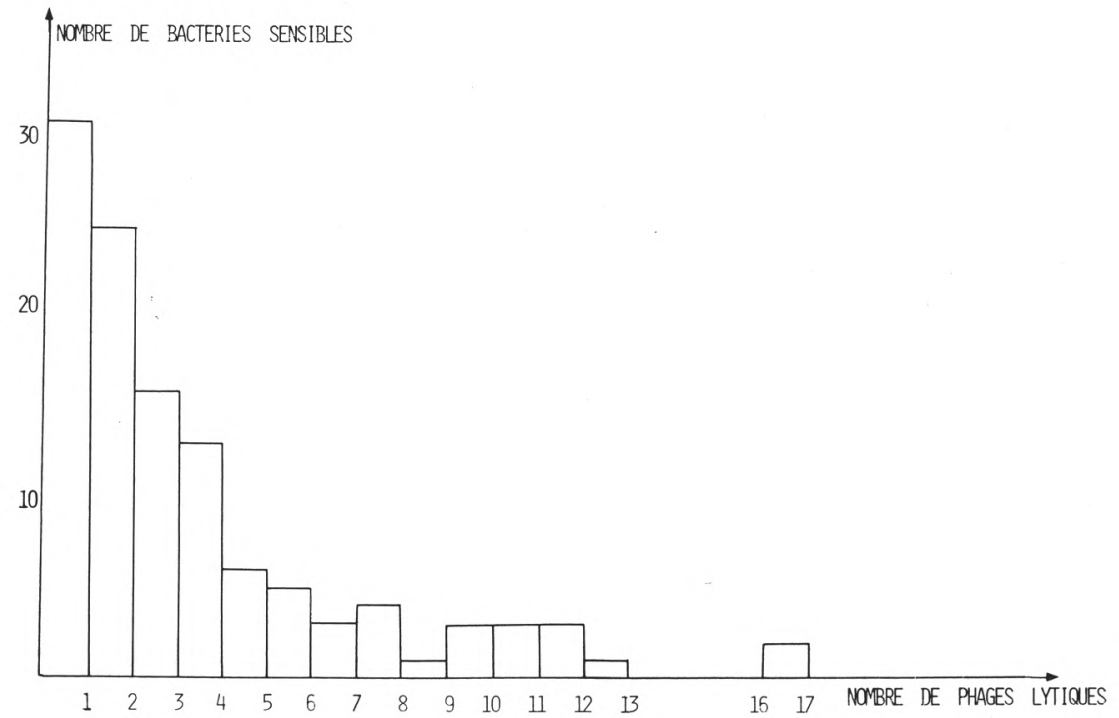

fig. 1

Histogramme illustrant la sensibilité des souches de Streptocoques lactiques à divers bactériophages

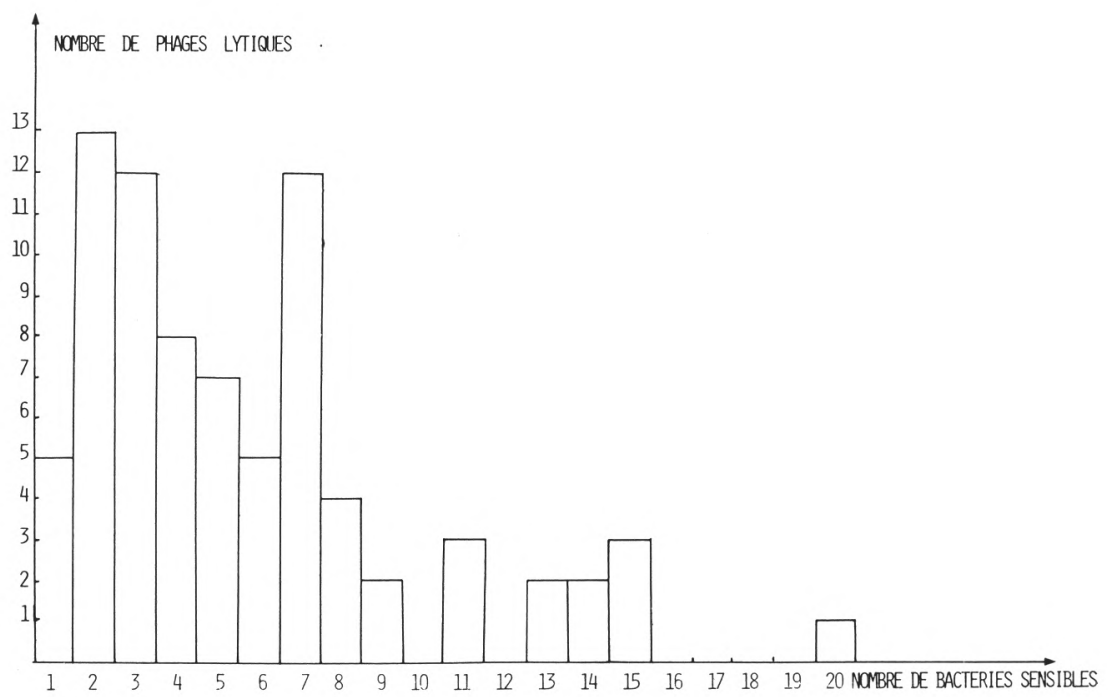

fig. 2

Histogramme illustrant le spectre d'action des phages lactiques. 


\section{TABLEAU 1}

Nombre de réactions positives obtenues avec 16 souches de Streptocoques lactiques

\begin{tabular}{|c|c|c|c|}
\hline Bactéries & $\begin{array}{c}\text { Souches } \\
\mathrm{N}^{\circ}\end{array}$ & $\begin{array}{c}\text { Phages } \\
\text { de collection }\end{array}$ & $\begin{array}{l}\text { Sérums } \\
\text { de fromagerie }\end{array}$ \\
\hline Streptococcus cremoris & $\begin{array}{l}1 \\
2 \\
3 \\
4 \\
5\end{array}$ & $\begin{array}{r}19 \\
4 \\
0 \\
2 \\
0\end{array}$ & $\begin{array}{r}9 \\
4 \\
1 \\
0 \\
12\end{array}$ \\
\hline Streptococcus diacetylactis & $\begin{array}{l}1 \\
2\end{array}$ & $\begin{array}{l}0 \\
7\end{array}$ & $\begin{array}{r}1 \\
36\end{array}$ \\
\hline Streptococcus lactis & $\begin{array}{l}1 \\
2 \\
3 \\
4 \\
5 \\
6 \\
7 \\
8 \\
9\end{array}$ & $\begin{array}{l}8 \\
0 \\
4 \\
1 \\
9 \\
4 \\
0 \\
0 \\
0\end{array}$ & $\begin{array}{r}4 \\
0 \\
0 \\
1 \\
33 \\
48 \\
0 \\
0 \\
1\end{array}$ \\
\hline
\end{tabular}

l'extérieur grâce aux enzymes de type muralytique contenues au niveau de la "queue" du phage. Des antibiotiques ou autres inhibiteurs présents dans les sérums pourraient donner les mêmes résultats.

En comparant les groupes de lysotypie obtenus par Leclerc [4], avec l'activité des sérums de fromagerie, il est possible de conclure que nous avons récupéré dans la nature des bactériophages appartenant aux groupes VI, VIII, IX, X. La majorité des réactions positives avec les sérums de fromagerie se retrouvent pour certaines souches utilisées en fromagerie dans les groupes VIII et IX.

\section{DISCUSSION ET CONCLUSIONS}

Une comparaison serait nécessaire entre tous les groupes de lysotypie définis. Cela impliquerait à l'échelle internationale un échange systématique des collections de bactéries et de bactériophages. 
La sensibilité des souches est très variable puisque, si 32 souches ne sont sensibles qu'à un seul phage, 25 le sont à deux virus, 16 à 3 , 13 à 4 ; et 2 souches se sont révélées sensibles à 17 phages différents [1].

La spécificité d'action des phages est également très variable, puisque 5 virus n'attaquent qu'une souche, 13 en attaquent 2, 12 en lysent 3 et un phage peut détruire 20 cultures différentes.

Certaines souches de streptocoques lactiques mésophiles se sont montrées non typables avec les phages de notre collection [4]. Nous avons donc recherché, pour compléter les tests de lysotypie, les virus susceptibles d'être dans les lactosérums de fromagerie. Ce travail devait permettre d'augmenter la validité de notre collection pour tester la sensibilité des souches bactériennes utilisées en industrie.

En comparant tous les tests de lysotypie, il est possible d'identifier les phages d'un très grand nombre de sérums aux groupes VI, VIII, IX et X définis par Leclerc [4].

Notre collection de phages ( +1 phage isolé de lactosérum) permet de tester d'une manière très représentative la sensibilité d'un grand nombre de souches.

Seules, trois variétés de streptocoques se sont révélées résistantes à l'ensemble des bactériophages de collection ou provenant de sérums. Ces cultures sont évidemment intéressantes en tant que levains, mais elles posent aussi le problème de l'origine de la résistance chez les streptocoques mésophiles. De toute manière, une étude plus systématique permettrait peut-être de mettre en évidence des sérums actifs contre ces bactéries.

L'élaboration des groupes de lysotypie pose le problème des mécanismes impliqués dans la sensibilité ou la résistance des streptocoques lactiques. Plusieurs hypothèses sont, en effet, envisageables :

- les groupes de lysotypie peuvent correspondre à des groupes d'adsorption ;

- ces groupes pourraient être mis en parallèle avec des systèmes de modification et restriction bien connus chez de nombreuses bactéries [13] ;

- enfin le phénomène de lysogénie pourrait interférer avec la distribution des cultures dans les groupes de lysotypie.

\section{Remerciements}

Nous tenons à remercier la Société Air-Liquide pour son aide intellectuelle, matérielle et technique. 


\section{S u m m a ry}

\section{LYSOTYPY AND BACTERIOPHAGE RESISTANCE OF LACTIC MESOPHILE SREPTOCOCCI}

164 strains of lactic mesophile Streptococci have been lysotyped by 79 bacteriophages. 10 lysotypic groups are defined. One bacteriophage lyses $S$. lactis. S. diacetylactis and $S$. cremoris.

69 wheys lyse 15 strains of industrial lactic mesophile streptococci. Bacteriophages classification of these french wheys are group VI, VIII, IX, X. Three strains are resistant against all the bacteriophages.

Reçu pour publication en octobre 1980.

\section{Bibliographie}

[1] Bozio (J. Y.), Leclerc (M. C.) et Guy (Ph.) (1975). - Etude du typage phagique de souches de bactéries lactiques destinées à l'industrie laitière. Rev. Lait. Fse, 334, p. 1-3.

[2] Chevalier (R.) (1972). - Recherches laitières effectuées au laboratoire de recherches de la Chaire de Microbiologie de 1'Institut National Agronomique Paris-Grignon. Rev. Lait. Fse, 297, 305-307.

[3] Diday (S.) (1971). - Une nouvelle méthode en classification automatique et reconnaissance des formes: la méthode des nuées dynamiques. Rev. Stat. Appl., 19, 19-33.

[4] Heap (H. A.), Limsowtin (G. K.) and Lawrence (R. C.) (1978). - Contribution of St. lactis strains in raw milk to phage infection in commercial cheese factories. N. Z. Jl. Dairy Science Technol., 13, 16-22.

[5] JARVIS (A. W.) (1978). - Serological studies of a host range mutant of a lactic Streptococcal bacteriophage. Appl. Environm. Microbiol., 36, 785-789.

[6] LAWRENCE (R. C.) (1978). - Action of bacteriophage on lactic acid bacteria : consequences and protection. N.Z. Jl. Dairy Sci. Technol., 13, 129-136.

[7] Leclerc (M. C.) (1976). - Contrôle des levains lactiques pour l'industrie laitière. Thèse de Doctorat $3^{\mathrm{e}}$ cycle, Université de Clermont II.

[8] Limsowtin (G. K. Y.), Heap (H. A.), Lawrence (R. C.) (1978). - Heterogenicity among strain of lactic Streptococci. N.Z. Jl. Dairy Sci. Technol., $13,1-8$.

[9] Mac Kay (L. L.), Cords (B. R.), Balivius (K. A.) (1973). - Transduction of lactose metabolism in Streptococcus lactis, $\mathrm{D}_{2}$. J. Bact., 116, 810-815.

[10] Sandine (W. E.) (1976). - New techniques in handling lactic cultures to enhance their performance. J. Dairy Science, 60, 822-828.

[11] Sozzi (T.), Poulin (J. M.), Maret (R.), Ponsaz (R.) (1978). - Isolation and some characteristics of phages of ropy strains of Streptococcus lactis. Milchwissenschaft, 33, 349-352.

[12] Sozzi (T.), Poulin (J.M.), Maret (R.) (1978). - Effect of incubation temperature on the development of lactic acid bacteria and their phages. J. Dairy Research, 45, 259-265.

[13] Winckler (K. C.) and Grootsen (Ca.) (1961). - The relation of phage pattern and lysogenicity in the phage typing of Staphylococci of phage group. II. J. Genet. Microbiol., 27, 225-246. 